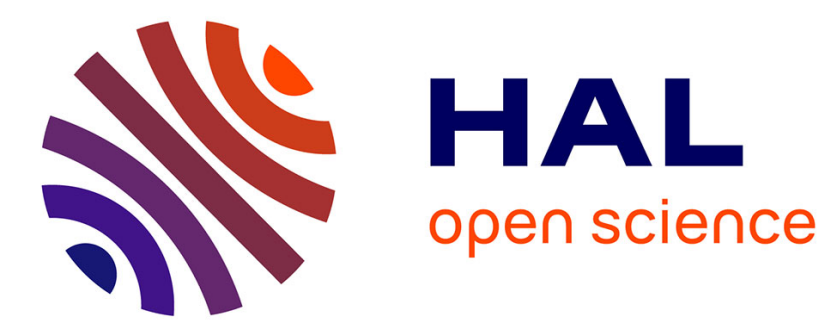

\title{
Influence de l'expertise sur l'évolution de la cadence de pédalage au cours d'un test progressif maximal
}

Sébastien Argentin, Christophe Hausswirth, François Hug, François Bieuzen, Jeanick Brisswalter

\section{To cite this version:}

Sébastien Argentin, Christophe Hausswirth, François Hug, François Bieuzen, Jeanick Brisswalter. Influence de l'expertise sur l'évolution de la cadence de pédalage au cours d'un test progressif maximal. Science \& Sports, 2006, 21 (5), pp.273 - 279. 10.1016/j.scispo.2006.08.002 . hal-01762787

\section{HAL Id: hal-01762787 \\ https://hal-insep.archives-ouvertes.fr/hal-01762787}

Submitted on 10 Apr 2018

HAL is a multi-disciplinary open access archive for the deposit and dissemination of scientific research documents, whether they are published or not. The documents may come from teaching and research institutions in France or abroad, or from public or private research centers.
L'archive ouverte pluridisciplinaire HAL, est destinée au dépôt et à la diffusion de documents scientifiques de niveau recherche, publiés ou non, émanant des établissements d'enseignement et de recherche français ou étrangers, des laboratoires publics ou privés. 


\title{
Influence de l'expertise sur l'évolution de la cadence de pédalage au cours d'un test progressif maximal
}

\section{Influence of expertise on the cycling cadence evolution during a maximal continuous incremental test}

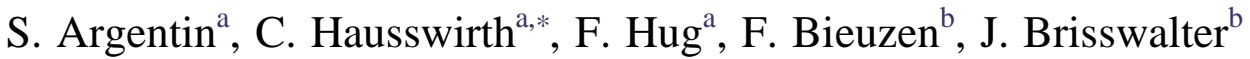 \\ ${ }^{a}$ Laboratoire de biomécanique et physiologie, institut national du sport et de l'éducation physique, avenue du Tremblay, 75012 Paris, France \\ ${ }^{\mathrm{b}}$ Laboratoire d'ergonomie sportive et de performance, EA 3162, université de Toulon-Var, avenue de l'Université, BP 132, 83957 La Garde cedex, France
}

Résumé

Objectifs. - L'objectif de ce travail est d'étudier la cadence librement choisie (CLC) au cours d'une épreuve de pédalage dite «triangulaire » chez différentes populations de sujets disposant d'aptitudes physiques et physiologiques différentes.

Méthodes. - Trois groupes de huit sujets (sportifs occasionnels, OCC - cyclistes régionaux, nationaux, CYC - coureurs régionaux, COU) ont participé à cette expérimentation. Il leur a été demandé de réaliser, lors d'une même session d'évaluation, un test de détermination de force maximale dynamique des membres inférieurs suivi, une heure plus tard, d'un test progressif, maximal, continu de détermination de la consommation maximale d'oxygène $\left(\mathrm{V}_{2 \max }\right)$ sur ergocycle à frein électromagnétique.

Résultats. - Nos résultats mettent en évidence l'adoption d'une CLC constante chez les cyclistes alors que les non cyclistes (i.e. OCC et COU) augmentent significativement leur CLC entre le début et la fin du test. En outre, bien que la CLC des cyclistes soit significativement plus élevée que celle des non cyclistes en début d'exercice, il n'existe ensuite plus aucune différence significative entre la CLC des trois populations.

Conclusion. - La stabilité de CLC des cyclistes peut être expliquée par l'expertise, c'est-à-dire, par le développement d'habiletés spécifiques induites par un processus d'apprentissage.

Abstract

Aim. - The purpose of this work is the study of the evolution of the freely chosen cadence (FCC) during a pedalling test, applied to various populations with different physical and physiological capacities.

Materials and methods. - Three groups of 8 subjects (less-trained non-cyclists, OCC - trained cyclists, CYC - trained runners, COU) participated in this experimentation. During a same session of evaluation, each subject realized a test of determination of the maximal dynamic strength of the lower limbs. This test was followed 1 hour later by a progressive, maximal and continuous test for determination of maximal oxygen uptake $\left(\mathrm{V}_{2 \max }\right)$ on an electromagnetic brake-cycle ergometer.

Results. - Our results show that trained cyclists adopt a constant FCC, while non-cyclists (i.e. OCC and COU) significantly increase their FCC between the beginning and the end of the test. Besides, although the FCC of the cyclists is significantly higher than the FCC of the noncyclists at the beginning of the exercise, one does not see any persisting difference between the FCC of the three populations, later on.

Conclusion. - The stability of cyclists' FCC can be explained through expertise, that is, throw the fact that the subjects develop specific skills inferred by a learning process.

Mots clés : Consommation maximale d'oxygène ; Cyclistes ; Athlètes ; Ergocycle ; Habiletés

Keywords: Maximal oxygen uptake; Cyclists; Runners; Cycle ergometer; Skill

\footnotetext{
*Auteur correspondant.

Adresse e-mail : christophe.hausswirth@insep.fr (C. Hausswirth).
} 


\section{Introduction}

Les critères de choix de la cadence de pédalage en cyclisme sont multiples et dépendent de l'interaction entre le niveau d'aptitude physique et physiologique du sujet et les contraintes de l'activité. Plusieurs études montrent que la cadence librement choisie (CLC) peut être influencée par le niveau d'aptitude aérobie du sujet [15] et son habileté gestuelle [4,8], mais également par la durée [32] et l'intensité d'exercice [16]. Pour une intensité d'exercice donnée, en fonction du choix de la cadence, l'athlète a la possibilité de minimiser soit la quantité d'énergie métabolique utilisée [2,4,27], soit l'activité musculaire $[13,30,34]$ ou la force appliquée sur les pédales [21].

De nombreuses études descriptives ont pu mettre en évidence pour une puissance de sortie donnée l'existence d'une relation curvilinéaire entre la consommation d'oxygène $\left(\mathrm{V}_{2 \max }\right)$ et la cadence de pédalage permettant de déterminer un coût énergétique optimal [3,4,14,15,27,30,31]. Ainsi, la cadence énergétiquement optimale (CEO), c'est-à-dire la cadence de pédalage pour laquelle le coût énergétique représenté par la consommation d'oxygène est minimale, se situe autour de $60 \mathrm{rpm}$ et ce, quel que soit l'exercice physique pratiqué ou le niveau d'entraînement. Les premières études s'intéressant à l'influence de l'intensité d'exercice sur les optima énergétiques indiquent une augmentation des valeurs de CEO avec l'élévation de la puissance de sortie [1,4,27]. Dans cette perspective, les travaux de Coast et Welch [4] mettent en évidence, chez cinq cyclistes, des optima cardiorespiratoires (i.e. dépense énergétique et fréquence cardiaque) à chaque puissance $(100,150,200,250$ et $300 \mathrm{~W})$ d'un test progressif. La CEO observée se déplace ainsi de 50 à 78 rpm lorsque la puissance de sortie varie respectivement de 100 à $300 \mathrm{~W}$.

Néanmoins, les valeurs de CLC de cyclistes entraînés sont systématiquement supérieures à ces valeurs de cadence les plus économiques [4,8,12,14,15,30-32]. Des valeurs de CLC supérieures à $80 \mathrm{rpm}$ sont effectivement fréquemment observées, que ce soit lors d'un record de l'heure sur vélodrome (CLC > $100 \mathrm{rpm}$ ) [25], d'un contre-la-montre sur route (CLC d'environ $100 \mathrm{rpm}$ ) [12], d'étapes de plat sur route [12] ou bien lors d'épreuves rectangulaires réalisées sur ergocycle $[2,9,11$, 15,26,30,32-34].

Plusieurs hypothèses sont avancées dans la littérature pour expliquer ce décalage entre CLC et CEO. Alors que certains auteurs incriminent l'aptitude aérobie des sujets [14,15], d'autres relient le choix de la CLC aux contraintes biomécaniques du pédalage. Ainsi, certains travaux mettent en évidence une diminution de la force résultante appliquée sur les manivelles par l'adoption de cadences élevées [21,31]. Il est à noter que la CLC évolue également en fonction de la durée [2,9,32] et de l'intensité de l'exercice [15]. Ainsi, les travaux de Marsh et Martin [15] montrent une diminution de la CLC avec l'augmentation de la puissance de sortie chez des sujets sédentaires alors qu'elle reste stable chez les sujets entraînés (i.e. cyclistes et athlètes).

La variation de CLC avec l'intensité d'exercice n'est cependant investiguée dans l'ensemble de ces études qu'au travers d'exercices réalisés à intensités constantes. À notre connaissance, il n'existe à ce jour aucun travail expérimental visant à décrire l'ajustement de la cadence de pédalage au cours d'un exercice continu et progressif (épreuve «triangulaire ») mené jusqu'à épuisement au cours duquel le stress métabolique augmente linéairement aux contraintes mécaniques. À la vue de la littérature déjà existante, nous pouvons supposer que la cadence varie en fonction de l'intensité de l'exercice pour s'adapter à la demande métabolique et/ou mécanique. L'objectif de notre travail sera donc d'étudier l'évolution de cette cadence librement choisie au cours d'une épreuve de pédalage triangulaire chez différentes populations de sujets disposant d'aptitudes physiques et physiologiques différentes (i.e. sportifs occasionnels, cyclistes et coureurs à pied).

\section{Méthodes}

\subsection{Sujets}

Vingt-quatre sujets masculins répartis en trois groupes ont participé à cette étude :

- huit sportifs occasionnels pratiquant une activité physique hebdomadaire de type aérobie à raison de 3,1 $\pm 0,8$ heures par semaine (OCC : âge : 31,7 $\pm 9,5$ ans, taille : $176,6 \pm 4,7 \mathrm{~cm}$, masse : 71,9 $\pm 9,7 \mathrm{~kg}$ ) ;

- huit cyclistes participant régulièrement à des compétitions de niveau interrégional et national (CYC : âge : $30,6 \pm 11,1$ ans ; taille : 176,1 $\pm 6,1 \mathrm{~cm}$; masse : $67,9 \pm 6,9 \mathrm{~kg})$;

- huit coureurs, pratiquant principalement sur des distances supérieures ou égales au marathon et de niveau régional (COU : âge : $36,3 \pm 7,6$ ans ; taille : $176,8 \pm 4,2 \mathrm{~cm}$; masse : $71,8 \pm 6,2 \mathrm{~kg})$.

Tous ces sujets étaient préalablement informés des procédures d'évaluation concernant les différents tests. Ils étaient volontaires et motivés par la participation à l'expérimentation. Le projet a été approuvé par le comité d'éthique local de SaintGermain-en-Laye, France.

\subsection{Protocole expérimental}

Lors d'une même session d'évaluation, tous les sujets ont effectué deux tests espacés d'une heure de récupération. Le premier test (test 1) consistait en la détermination du niveau de force maximale dynamique $\left(\mathrm{F}_{\max }\right)$ lors d'un mouvement sollicitant préférentiellement les chaînes musculaires des extenseurs de hanche et des membres inférieurs, principalement sollicitées lors d'un exercice de pédalage [6]. Il était ensuite demandé aux sujets de réaliser une épreuve de détermination du potentiel maximal aérobie (test 2) sur bicyclette ergométrique à frein électromagnétique (type Excalibure, Lode, Groningen, Pays-bas). Afin de s'affranchir d'éventuelles fluctuations de la force produite lors des actions musculaires 
dynamiques au cours de la journée [7,23] tous ces tests ont été réalisés au même moment, entre 10 h 00 et 12 h 00 .

\subsection{1. Évaluation de la force maximale des extenseurs des membres inférieurs}

L'évaluation de la force maximale, représentée par la charge soulevée une seule et unique fois (1RM), était réalisée à partir d'une presse à membres inférieurs inclinée à $45^{\circ}$ (Presse Manuelle, Multi-form, La Roque d'Anthéor, France). La position des pieds sur la plate-forme était ajustée de manière à former un angle articulaire de $90^{\circ}$ entre la jambe et la cuisse. À partir de cette position initiale, les sujets devaient effectuer un mouvement d'extension des membres inférieurs durant lequel les conditions de réalisation étaient standardisées (écartement des pieds, amplitude gestuelle) et un investissement maximal était exigé. Préalablement à la session de test, les sujets ont effectué une session de familiarisation avec l'appareil et les techniques gestuelles spécifiques à celui-ci [28]. Ensuite, et après un échauffement standardisé, la procédure de détermination du niveau de $\mathrm{F}_{\max }$ consistait en plusieurs séries réalisées avec des charges progressivement augmentées jusqu'à l'atteinte du 1RM. La dernière charge soulevée avec succès une seule et unique fois était alors considérée comme le 1RM [17]. Chacune des répétitions était suivie d'une période de récupération de trois minutes.

\subsection{2. Épreuve de détermination de la puissance aérobie maximale}

Après une période d'échauffement de six minutes réalisées à $100 \mathrm{~W}$, la puissance était augmentée de $30 \mathrm{~W}$ toutes les minutes jusqu'à ce que le sujet ne puisse plus poursuivre l'exercice à la puissance imposée. Les athlètes étaient encouragés verbalement afin qu'ils poursuivent leur effort jusqu'à épuisement. La cadence de pédalage était librement choisie par les sujets sans qu'aucune rétroaction visuelle ne leur soit communiquée. Les échanges gazeux et respiratoires étaient enregistrés en cycle à cycle pendant toute la durée du test à l'aide d'un système télémétrique de type Cosmed $\mathrm{K} 4 \mathrm{~b}^{2}$ (Rome, Italie) et calibré préalablement à chacun des tests. La fréquence cardiaque a été enregistrée en continu pendant toute la durée du test grâce à un cardiofréquencemètre (POLAR S625X, Finlande).

\subsubsection{Recueil des paramètres ventilatoires et gazeux}

Les paramètres étudiés lors du test sur bicyclette à charge croissante étaient le débit de consommation d'oxygène ( $\mathrm{V} \mathrm{O}_{2 \max }$, en $1 \mathrm{~min}^{-1}$ et $\mathrm{ml} \mathrm{kg}^{-1} \min ^{-1}$ ), le débit ventilatoire $\left(\mathrm{V}_{\mathrm{E}}\right.$, en $\left.1 \mathrm{~min}^{-1}\right)$, la fréquence respiratoire $(\mathrm{FR}$, en cycles $\min ^{-1}$ ) ainsi que la fréquence cardiaque (FC, en batt $\min ^{-1}$ ). Les valeurs maximales ainsi que les valeurs correspondantes aux deux seuils ventilatoires $\left(\mathrm{SV}_{1}\right.$ et $\left.\mathrm{SV}_{2}\right)$ ont été prises en compte.

Le premier et le second seuils ventilatoires (respectivement $\mathrm{SV}_{1}$ et $\mathrm{SV}_{2}$ ) ont été déterminés simultanément par plusieurs évaluateurs d'après la méthode proposée par Reinhard et al. [24] reposant sur l'identification de cassures dans l'évolution de l'équivalent ventilatoire pour l'oxygène $\left(\mathrm{V}_{\mathrm{E}} / \mathrm{V} \mathrm{O}_{2}\right)$ et l'équivalent respiratoire pour le gaz carbonique $\left(\mathrm{V}_{\mathrm{E}} / \mathrm{V} \mathrm{CO}_{2}\right)$.

Les critères d'atteinte de $\mathrm{V} \mathrm{O}_{2 \max }$ étaient un plafonnement de $\mathrm{V} \mathrm{O}_{2}$ dans les derniers paliers (moins de $2 \mathrm{ml} \mathrm{kg}^{-1} \mathrm{~min}^{-1}$ d'incrément entre deux paliers), une fréquence cardiaque à l'épuisement égale à la fréquence cardiaque maximale théorique $(220$ - âge $) \pm 10$ batt $\min ^{-1}$ et un quotient respiratoire supérieur à 1,10 [10]. $\vee \mathrm{O}_{2 \max }$ était alors définie comme la valeur moyenne sur 30 secondes au plateau.

\subsubsection{Variables mécaniques}

Plusieurs variables mécaniques étaient enregistrées en continu lors du test : la cadence de pédalage, le couple maximal $\left(\mathrm{C}_{\max }\right)$ et minimal $\left(\mathrm{C}_{\min }\right)$ exercé sur chacune des pédales, ainsi que l'angle pour lequel le couple maximal est obtenu par rapport à l'axe vertical. Le point initial du référentiel utilisé pour les mesures d'angles correspond à la position verticale haute de la manivelle droite. En l'absence de différence significative entre les valeurs enregistrées pour la pédale droite et gauche, nous avons choisi de moyenner, pour chacune des variables, les valeurs des deux pédales afin de simplifier la lecture de nos résultats. Pour chacune de ces variables mécaniques, nous avons retenu à chaque palier d'effort d'une minute, la moyenne des valeurs enregistrées entre la $20^{\mathrm{e}}$ et la $50^{\mathrm{e}}$ seconde du palier. Afin de rendre compte de l'évolution de ces variables, nous avons retenu les valeurs de début d'exercice $(100 \mathrm{~W})$, celles associées à $\mathrm{SV}_{1}$ et $\mathrm{SV}_{2}$ ainsi que celles de fin de test (à l'exception du dernier palier). Par ailleurs, la variation de ces variables mécaniques entre chacun de ces instants identifiés ci-dessus était prise en compte pour chaque sujet et exprimée en pourcentage de variation.

\subsubsection{Traitement statistique}

Tous les résultats sont exprimés sous la forme moyenne \pm écart-type. La normalité des différentes distributions a été testée à l'aide d'un test de Kolmogorov-Smirnov. La comparaison entre les différents indices obtenus par les trois groupes a ensuite été réalisée à partir d'une analyse de variance (Anova) pour des échantillons indépendants. Une analyse de variance à mesures répétées permettait d'identifier un effet de la période d'analyse sur les différentes variables. Dans le cas où apparaissait un effet significatif de la période, les différences entre chaque période étaient ensuite détectées à partir d'un test post-hoc de Newman-Keuls. Un test de corrélation (Spearman) nous a permis de calculer les coefficients de corrélation entre le niveau de $\mathrm{F}_{\max }$ des membres inférieurs et les différentes variables physiologiques et mécaniques enregistrées au cours du test. Le seuil de significativité a été fixé pour l'ensemble de nos analyses statistiques à $p<0,05$.

\section{Résultats}

Les paramètres physiques et physiologiques mesurés au cours du test progressif sont présentés dans le Tableau 1. 
Tableau 1

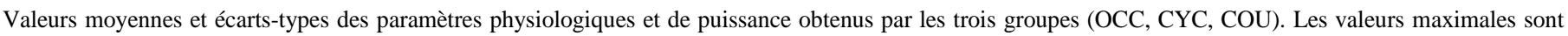
représentées ainsi que les valeurs obtenues aux premier et deuxième seuils ventilatoires $\left(\mathrm{SV}_{1}\right.$ et $\left.\mathrm{SV}_{2}\right)$

\begin{tabular}{|c|c|c|c|c|}
\hline & & OCC (nombre = 8) & COU (nombre = 8) & CYC (nombre $=8$ ) \\
\hline \multirow[t]{5}{*}{ Valeurs maximales } & $P_{\max }$ (watts) & $310 \pm 31$ & $332 \pm 28$ & $415 \pm 21^{\mathrm{a}, \mathrm{b}}$ \\
\hline & $\mathrm{VO}_{2 \max }\left(1 \mathrm{~min}^{-1}\right)$ & $3,5 \pm 0,5^{\mathrm{b}}$ & $4,0 \pm 0,3^{\mathrm{a}}$ & $4,6 \pm 0,3^{\mathrm{a}, \mathrm{b}}$ \\
\hline & $\mathrm{VO}_{2 \max }\left(\mathrm{ml} \mathrm{kg}^{-1} \mathrm{~min}^{-1}\right)$ & $49,5 \pm 6,1^{\mathrm{b}}$ & $56,7 \pm 5,9^{\mathrm{a}}$ & $67,5 \pm 5,4^{a, b}$ \\
\hline & $\mathrm{FC}_{\max }\left(\right.$ batt $\left.\min ^{-1}\right)$ & $184 \pm 13$ & $174 \pm 6$ & $185 \pm 12$ \\
\hline & $\mathrm{V}_{\mathrm{Emax}}\left(1 \min ^{-1}\right)$ & $144,6 \pm 32,8$ & $153,5 \pm 23,8$ & $172,0 \pm 30,6$ \\
\hline \multirow[t]{6}{*}{ Valeurs à $S V_{2}$} & $\mathrm{P}$ (watts) & $232 \pm 25^{b}$ & $264 \pm 31^{\mathrm{a}}$ & $329 \pm 23^{\mathrm{a}, \mathrm{b}}$ \\
\hline & $\mathrm{P}\left(\%\right.$ de $\left.\mathrm{P}_{\max }\right)$ & $74,8 \pm 2,4$ & $79,5 \pm 5,8$ & $79,3 \pm 4,9$ \\
\hline & $\mathrm{VO}_{2}\left(\mathrm{ml} \mathrm{kg}{ }^{-1} \min ^{-1}\right)$ & $39,9 \pm 3,9^{b}$ & $47,9 \pm 6,5^{\mathrm{a}}$ & $57,3 \pm 4,4^{\mathrm{a}, \mathrm{b}}$ \\
\hline & $\mathrm{VO}_{2}\left(\%\right.$ de $\left.\mathrm{VO}_{2 \max }\right)$ & $80,8 \pm 3,5$ & $84,3 \pm 3,7$ & $84,9 \pm 3,1$ \\
\hline & $\mathrm{FC}\left(\right.$ batt $\left.\min ^{-1}\right)$ & $169 \pm 15^{b}$ & $154 \pm 11^{\mathrm{a}}$ & $172 \pm 12^{b}$ \\
\hline & $\mathrm{FC}\left(\%\right.$ de $\left.\mathrm{FC}_{\max }\right)$ & $91,7 \pm 4,6$ & $88,6 \pm 4,3$ & $93,3 \pm 2,4$ \\
\hline \multirow[t]{6}{*}{ Valeurs à $S V_{1}$} & $\mathrm{P}$ (watts) & $161 \pm 16$ & $179 \pm 18$ & $231 \pm 19^{\mathrm{a}, \mathrm{b}}$ \\
\hline & $\mathrm{P}\left(\%\right.$ de $\left.\mathrm{P}_{\max }\right)$ & $52,2 \pm 3,6$ & $53,8 \pm 4,1$ & $55,7 \pm 3,4$ \\
\hline & $\mathrm{VO}_{2}\left(\mathrm{ml} \mathrm{kg}^{-1} \min ^{-1}\right)$ & $29,5 \pm 2,9^{b}$ & $36,5 \pm 4,2^{\mathrm{a}}$ & $46,3 \pm 3,8^{\mathrm{a}, \mathrm{b}}$ \\
\hline & $\mathrm{VO}_{2}\left(\%\right.$ de $\left.\mathrm{VO}_{2 \max }\right)$ & $59,7 \pm 2,7$ & $64,4 \pm 4,7$ & $68,8 \pm 6,0^{\mathrm{a}}$ \\
\hline & $\mathrm{FC}\left(\right.$ batt $\left.\mathrm{min}^{-1}\right)$ & $149 \pm 22^{b}$ & $130 \pm 13^{\mathrm{a}}$ & $152 \pm 13^{\mathrm{b}}$ \\
\hline & $\mathrm{FC}\left(\%\right.$ de $\left.\mathrm{FC}_{\max }\right)$ & $80,5 \pm 8,6$ & $74,6 \pm 6,7$ & $82,1 \pm 4,4$ \\
\hline
\end{tabular}

Seuil de significativité : $p=0,05$.

a Différence significative avec le groupe OCC.

b Différence significative avec le groupe COU.

\subsection{Force maximale}

Les valeurs de Fmax (en $\mathrm{kg}$ ) mesurées sur la presse inclinée sont respectivement de $265,1 \pm 26,1 \mathrm{~kg} ; 286,0 \pm 27,9 \mathrm{~kg}$ et de $263,0 \pm 39,2 \mathrm{~kg}$ pour les groupes OCC, CYC et COU. Aucune différence significative de niveau de force n'apparaît entre ces groupes. Ce niveau de force maximal des membres inférieurs est toutefois significativement corrélé à Pmax sur ergocycle pour les non-cyclistes $(r=0,63 ; n=16 ; p<0,05)$.

\subsection{Paramètres mécaniques}

L'Anova montre un effet période pour les valeurs de cadence de pédalage. En début d'exercice, les cadences adoptées par les cyclistes $(92,6 \pm 12,4 \mathrm{rpm})$ sont significativement supérieures à celles adoptées par les sportifs occasionnels $(74,1 \pm 11,8 \mathrm{rpm})$ et les coureurs $(75,9 \pm 16,0 \mathrm{rpm})$. L'évolution de cette cadence de pédalage au cours du test est différente selon les groupes (Fig. 1). La cadence spontanément adoptée par les cyclistes en début d'exercice reste constante durant tout le test. À l'inverse, la cadence plus faible de début d'exercice des sportifs occasionnels et des coureurs présente une tendance à l'augmentation tout au long de l'exercice. Cette augmentation de cadence n'est toutefois significative qu'entre les valeurs de début et celles observées à $\mathrm{SV}_{2}(+7,5 \pm 6,5 \%$ pour $\mathrm{OCC}$ et $+9,6 \pm 13,2 \%$ pour COU ; $p<0,05)$ et en fin d'exercice (+ $12,9 \pm 11,0 \%$ pour OCC et $+11,8 \pm 15,9 \%$ pour $\mathrm{COU}$; $p<0,05)$. À $\mathrm{SV}_{1}, \mathrm{SV}_{2}$ et en fin d'exercice, les cadences de pédalages des cyclistes ne sont plus statistiquement différentes de celles des sportifs occasionnels et des coureurs et d'importantes différences interindividuelles apparaissent au sein de chaque groupe.

Les autres variables mécaniques enregistrées au cours du test progressif sont présentées dans le Tableau 2. Il n'existe aucune différence significative entre les groupes pour les indi-

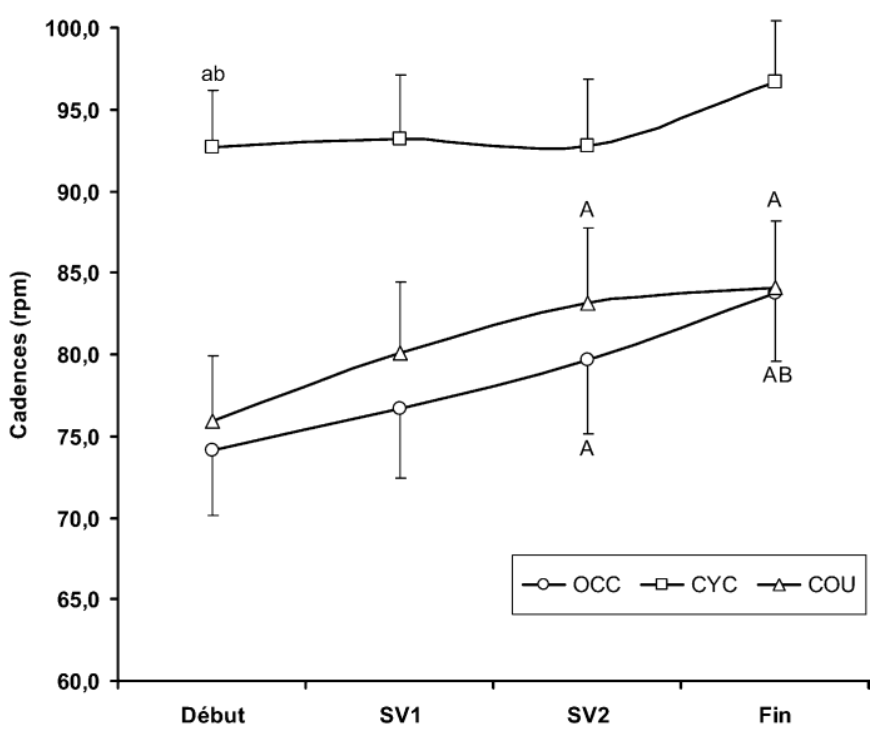

Fig. 1. Evolution de la cadence de pédalage au cours du test progressif, maximal pour les 3 groupes (OCC, COU et CYC).

${ }^{a}$ Différence significative avec le groupe OCC.

${ }^{\mathrm{b}}$ Différence significative avec le groupe COU.

${ }^{A}$ Différence significative avec Début.

${ }^{\mathrm{B}}$ Différence significative avec $\mathrm{SV}_{1}$.

${ }^{\mathrm{C}}$ Différence significative avec $\mathrm{SV}_{2}$.

Seuil de significativité : $p=0,05$.

ces $\mathrm{C}_{\max }, \mathrm{C}_{\min }$ et angle à chacune des périodes d'analyse considérées. Afin de comparer ces variables à des puissances de sortie identiques, une analyse supplémentaire a également été effectuée pour l'ensemble des trois groupes à 160 et $220 \mathrm{~W}$, celle-ci ne présentant toujours pas de différences significatives entre nos trois groupes.

Concernant l'évolution de ces variables au cours du test, les valeurs de $\mathrm{C}_{\max }$ sont directement reliées à l'augmentation de la puissance de sortie au cours du test pour tous les groupes : 
Tableau 2

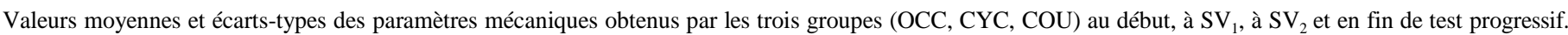

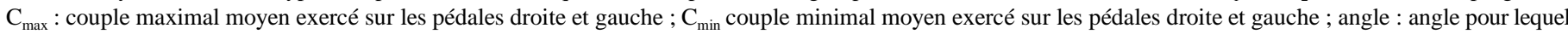
le couple maximal est obtenu par rapport à l'axe vertical

\begin{tabular}{|c|c|c|c|c|}
\hline & & OCC $($ nombre $=8)$ & COU (nombre = 8) & CYC (nombre $=8$ ) \\
\hline \multirow[t]{4}{*}{ Valeurs de Début } & Cadence (cycles.min ${ }^{-1}$ ) & $74,1 \pm 11,8$ & $75,9 \pm 16,0$ & $92,6 \pm 12,4^{\mathrm{a}, \mathrm{b}}$ \\
\hline & $\mathrm{C}_{\max }(\mathrm{Nm})$ & $38,5 \pm 6,2$ & $37,2 \pm 6,2$ & $35,2 \pm 3,2$ \\
\hline & $\mathrm{C}_{\min }(\mathrm{Nm})$ & $-8,4 \pm 2,7$ & $-7,8 \pm 3,8$ & $-11,1 \pm 6,5$ \\
\hline & Angle (Degrés) & $93,3 \pm 6,9$ & $95,9 \pm 10,7$ & $95,9 \pm 4,6$ \\
\hline \multirow[t]{4}{*}{ Valeurs à $S V_{l}$} & Cadence (cycles. $\left.\min ^{-1}\right)$ & $76,7 \pm 12,0$ & $80,1 \pm 18,3$ & $93,2 \pm 15,2$ \\
\hline & $\mathrm{C}_{\max }(\mathrm{Nm})$ & $45,2 \pm 4,0^{c}$ & $50,3 \pm 11,1^{\mathrm{c}}$ & $56,3 \pm 8,1^{\mathrm{a}, \mathrm{c}}$ \\
\hline & $\mathrm{C}_{\min }(\mathrm{Nm})$ & $-11,4 \pm 2,6^{\mathrm{c}}$ & $-10,6 \pm 2,8^{\mathrm{c}}$ & $-13,4 \pm 4,9$ \\
\hline & Angle (Degrés) & $90,9 \pm 5,6$ & $90,1 \pm 10,7$ & $94,6 \pm 5,1$ \\
\hline \multirow[t]{4}{*}{ Valeurs à $S V_{2}$} & Cadence (cycles.min ${ }^{-1}$ ) & $79,7 \pm 13,8^{c}$ & $83,2 \pm 20,7^{\mathrm{c}}$ & $92,8 \pm 17,0$ \\
\hline & $\mathrm{C}_{\max }(\mathrm{Nm})$ & $55,3 \pm 7,5^{\mathrm{c}, \mathrm{d}}$ & $64,7 \pm 15,0^{\mathrm{c}, \mathrm{d}}$ & $69,9 \pm 12,3^{\mathrm{c,d}}$ \\
\hline & $\mathrm{C}_{\min }(\mathrm{Nm})$ & $-9,4 \pm 2,6^{\mathrm{d}}$ & $-9,2 \pm 3,5$ & $-10,9 \pm 6,7$ \\
\hline & Angle (Degrés) & $86,8 \pm 3,8^{c}$ & $91,8 \pm 10,6$ & $89,2 \pm 5,9^{\mathrm{c}, \mathrm{d}}$ \\
\hline \multirow[t]{4}{*}{ Valeurs de fin } & Cadence (cycles. $\left.\min ^{-1}\right)$ & $83,7 \pm 17,3^{\mathrm{cd}}$ & $84,8 \pm 16,6^{c}$ & $96,7 \pm 14,0$ \\
\hline & $\mathrm{C}_{\max }(\mathrm{Nm})$ & $65,2 \pm 8,6^{\mathrm{c}, \mathrm{d}, \mathrm{e}}$ & $78,8 \pm 17,8^{\mathrm{c}, \mathrm{d}, \mathrm{e}}$ & $80,7 \pm 14,7^{\mathrm{c}, \mathrm{d}, \mathrm{e}}$ \\
\hline & $\mathrm{C}_{\min }(\mathrm{Nm})$ & $-8,4 \pm 2,7^{\mathrm{d}}$ & $-7,8 \pm 3,8^{\mathrm{d}}$ & $-11,1 \pm 6,5$ \\
\hline & Angle (Degrés) & $87,4 \pm 6,2^{c}$ & $88,5 \pm 9,9^{c}$ & $85,4 \pm 6,4^{\mathrm{c}, \mathrm{d}}$ \\
\hline
\end{tabular}

Seuil de significativité : $p=0,05$.

${ }^{a}$ Différence significative avec le groupe OCC.

${ }^{\mathrm{b}}$ Différence significative avec le groupe COU.

Différence significative avec début.

${ }^{d}$ Différence significative avec $\mathrm{SV}_{1}$.

e Différence significative avec $\mathrm{SV}_{2}$.

toutes les valeurs sont ainsi significativement différentes d'une période à l'autre. Les valeurs de l'angle pour lequel le couple maximal est obtenu par rapport à l'axe vertical présentent quant à elles une tendance générale allant dans le sens d'une diminution de celui-ci au cours du test. Cette diminution est significative pour nos trois groupes qu'entre les valeurs de début et de fin. Enfin, une stabilité du travail musculaire négatif lors de la phase de traction, reflétée par les valeurs de $\mathrm{C}_{\min }$, est observée tout au long du test pour les cyclistes.

\section{Discussion}

Cette étude rapporte pour la première fois l'évolution de la CLC chez trois groupes de sujets (i.e. CYC, OCC et COU) réalisant une épreuve de pédalage progressive, continue et maximale sur ergocycle. Nos résultats mettent en évidence l'adoption d'une CLC constante chez les cyclistes alors que les non-cyclistes (i.e. OCC et COU) augmentent significativement leur CLC entre le début et la fin du test. Par ailleurs, bien que la CLC des cyclistes soit significativement plus élevée que celle des non-cyclistes en début d'exercice, il n'existe ensuite plus aucune différence significative entre la CLC des trois populations.

La plupart des données disponibles dans la littérature montrent, dans le cadre d'exercices d'intensité constante, des CLC significativement plus élevées chez les cyclistes [5,8,22]. Toutefois, il semblerait que cela ne soit pas toujours le cas et plusieurs études ont observé des résultats contradictoires. Marsh et Martin [14] ont enregistré des CLC statistiquement similaires au cours d'un exercice sur ergocycle réalisé à une puissance de $200 \mathrm{~W}$ pour huit cyclistes et huit non-cyclistes, respectivement $85,2 \pm 9,2 \mathrm{rpm}$ et 91,6 $\pm 10,5 \mathrm{rpm}$. Ces mêmes auteurs ont plus récemment complété leurs observations dans une étude mettant également en évidence des CLC similaires chez des cyclistes et coureurs [15]. Au cours d'exercices effectués à des puissances comprises entre 75 et $250 \mathrm{~W}$, les cyclistes $\left(\vee \mathrm{O}_{2 \max }=70,7 \pm 4,1 \mathrm{ml} \mathrm{kg}^{-1} \mathrm{~min}^{-1}\right)$ et les coureurs à pied $\left(\vee \mathrm{O}_{2 \max }=72,5 \pm 2,2 \mathrm{ml} \mathrm{kg}^{-1} \mathrm{~min}^{-1}\right.$ ) présentaient des CLC statistiquement identiques et systématiquement supérieures à celles des sujets moins entraînés ( $\mathrm{V} \mathrm{O}_{2 \max }=44,2 \pm 2,8 \mathrm{ml} \mathrm{kg}^{-1} \mathrm{~min}^{-1}$ ), proches au niveau de leur potentiel aérobie de nos sujets du groupe OCC $\left(\vee \mathrm{O}_{2 \max }=49,5 \pm 6,1 \mathrm{ml} \mathrm{kg}^{-1} \mathrm{~min}^{-1}\right)$. Les deux groupes de sportifs entraînés conservaient une CLC comprise entre 90 et $100 \mathrm{rpm}$ indépendamment de la puissance de sortie alors que les sujets moins entraînés utilisaient des cadences significativement inférieures, allant d'environ $80 \mathrm{rpm}$ à $75 \mathrm{~W}$ à $65 \mathrm{rpm}$ lorsque la puissance montait jusqu'à $175 \mathrm{~W}$. À travers cette étude, ces auteurs suggèrent que l'aptitude aérobie serait un facteur déterminant dans le choix de la cadence de pédalage alors, qu'au contraire, l'expertise cycliste ne le serait pas. Cette hypothèse ne semble toutefois pas pouvoir s'appliquer dans notre cas. En effet, les trois populations de notre étude présentent des valeurs de CLC identiques que ce soit à $\mathrm{SV}_{1}, \mathrm{SV}_{2}$ ou en fin d'exercice, et ce malgré des différences significatives de $\mathrm{VO}_{2 \max }$. Cependant, la comparaison avec nos résultats est à effectuer avec précaution dans la mesure où le protocole de Marsh et Martin [15] consistait à réaliser des épreuves rectangulaires de huit minutes et que seules les six dernières minutes étaient étudiées. Ce contexte expérimental est différent du nôtre dont la singularité est d'étudier spécifiquement l'adaptation de la CLC à l'augmentation des contraintes métaboliques et mécaniques au cours d'une épreuve triangulaire. Par ailleurs, il est possible que la différence d'aptitude aérobie de notre population de coureurs et sportifs occa- 
sionnels $\left(7,2 \mathrm{ml} \mathrm{kg}^{-1} \mathrm{~min}^{-1}\right)$ ne soit pas suffisante pour induire des modifications de CLC.

Le résultat marquant de notre étude concerne l'évolution de la cadence de pédalage au cours du test triangulaire selon les groupes (Fig. 1). La cadence plus faible de début d'exercice des sportifs occasionnels et des coureurs augmente significativement entre le début et la fin de l'exercice. Cette évolution de CLC, rencontrée pour notre groupe de sportifs occasionnels lors de ce type de test, est opposée à celle relevée par Marsh et al. [16] et Marsh et Martin [15]. Toutefois, l'élévation de CLC observée dans notre étude avec l'incrément de puissance, indépendamment du potentiel aérobie, pourrait être expliquée par les travaux de Patterson et Moreno [21] et de Takaishi et al. [31]. Selon ces auteurs, une augmentation de la cadence de pédalage correspondrait à une diminution des forces appliquées sur les pédales et de ce fait permettrait de minimiser l'action musculaire à produire [18]. Nos sujets non cyclistes choisiraient donc d'augmenter leur CLC avec l'augmentation de la puissance de sortie afin de minimiser l'action musculaire croissante à produire. De ce fait, les sujets possédant les plus hauts niveaux de $\mathrm{F}_{\max }$ dynamique des membres inférieurs seraient en mesure d'atteindre de plus hautes valeurs de $\mathrm{P}_{\max }$ (corrélation significative observée entre le niveau de $\mathrm{F}_{\max }$ des membres inférieurs et la $\mathrm{P}_{\max }$ pour nos deux groupes non-cyclistes, OCC et COU $n=16 ; r=0,63 ; p<0,05)$. Cette augmentation de CLC par les non-cyclistes au cours de notre test progressif pourrait également s'expliquer par un recrutement progressif des unités motrices rapides [35] dont l'efficience augmente avec la cadence de pédalage [29].

À l'inverse, la cadence plus importante spontanément adoptée par les cyclistes en début d'exercice reste constante durant tout le test. Bien que ce résultat soit en accord avec les observations de Marsh et al. [16] qui ont constaté une stabilité de la CLC des cyclistes entraînés lors d'épreuves rectangulaires réalisées à différentes puissances, on peut se demander ce qui explique cette stabilité de cadence au cours de notre test où l'objectif est d'atteindre progressivement la puissance maximale aérobie. En effet, les contraintes métaboliques et mécaniques augmentent au cours de ce type d'épreuve avec la puissance d'exercice. Si l'on considère qu'en fonction du choix de la cadence de pédalage l'athlète peut minimiser soit la quantité d'énergie métabolique utilisée [4,27], soit l'activité musculaire $[13,30]$ ou la force appliquée sur les pédales [21], on aurait alors pu supposer que l'évolution des contraintes de l'activité implique une variation de la CLC au cours du test. Les valeurs de CLC mesurées chez les cyclistes à $\mathrm{SV}_{2}(92,8 \pm 17,0 \mathrm{rpm})$ et en fin de test $(96,7 \pm 14,0 \mathrm{rpm})$ sont très proches des valeurs des optima (mécanique et musculaire) précédemment rapportées pour des puissances élevées [18,31]. MacIntosh et al. [13], dans une étude s'intéressant à l'influence de la puissance de sortie sur la détermination d'un optimum neuromusculaire, ont effectivement mis en évidence que la cadence optimale correspondant à une minimisation de la somme des valeurs de EMG-RMS de sept des principaux muscles impliqués dans le mouvement cyclique de pédalage (biceps femoris, gluteus maximus, gastrocnemius, rectus femoris, soleus, tibialis anterior et vastus lateralis) était de $86 \mathrm{rpm}$ à $300 \mathrm{~W}$ et de $99 \mathrm{rpm}$ pour une puissance de sortie de $400 \mathrm{~W}$. Les cadences élevées associées aux hautes puissances de fin de test, et proches de celles rencontrées en situation de compétition, permettent ainsi aux cyclistes entraînés de minimiser l'activité musculaire et les forces appliquées sur les pédales [16,21], d'avoir un moindre niveau d'activation musculaire [31], de percevoir une sensation de confort plus importante liée aux moindres sensations de tension musculaire [20] et d'optimiser leur production de force au niveau articulaire lors du cycle de pédalage [19]. Nous pouvons donc supposer que nos cyclistes utilisent à la fin de l'exercice des cadences de pédalage élevées leur permettant d'avoir un certain confort musculaire et articulaire malgré les hautes puissances de sortie rencontrées. Les valeurs élevées de CLC observées également en début de test, aux puissances plus faibles, pourraient donc s'expliquer par le développement d'habiletés spécifiques de pédalage induites par un processus d'apprentissage. En d'autres termes, les cyclistes adopteraient lors des entraînements et compétitions des CLC proches des optima musculaires et mécaniques rapportés dans la littérature [13,18,30,31] impliquant l'apprentissage d'un patron de recrutement musculaire spécifique [31] qui serait automatiquement réutilisé à des intensités d'exercices plus faibles. Cette hypothèse semble confirmée par nos résultats mettant en avant une stabilité, chez les cyclistes, du travail musculaire négatif mis en jeu lors de la phase de traction et reflétée par les valeurs de $\mathrm{C}_{\min }$.

\section{Conclusion}

L'évolution de la CLC au cours d'un test progressif continu et maximal est différente selon l'expertise des sujets. La stabilité de CLC observée chez les cyclistes au cours de ce type de test triangulaire peut être expliquée par le développement d'habiletés spécifiques induites par un processus d'apprentissage au fur et à mesure de leurs entraînements.

\section{Références}

[1] Banister EW, Jackson EW. The effect of speed and load changes on oxygen intake for equivalent power outputs during bicycle ergometry. Arbeitsphysiologie 1967;24:284-90.

[2] Brisswalter J, Hausswirth C, Smith D, Vercruyssen F, Vallier JM. Energetically optimal cadence vs. freely-chosen cadence during cycling: effect of exercise duration. Int J Sports Med 2000;21(1):60-4.

[3] Chavaren J, Calbet JAL. Cycling efficency and pedalling frequency in road cyclists. Eur J Appl Physiol 1999;80:555-63.

[4] Coast JR, Welch HG. Linear increase in optimal pedal rate with increased power output in cycle ergometry. Eur J Appl Physiol Occup Physiol 1985;53(4):339-42.

[5] Drake G. The cadence question: is it time to modify the pedalfast prescription? Bicycling 1993;34:44-6.

[6] Ericson MO, Bratt A, Nisell R, Nemeth G, Ekholm J. Load moments about the hip and knee joints during ergometer cycling. Scand J Rehabil Med 1986;18(4):165-72.

[7] Gaulthier A, Davenne D, Matrin A, Van Hoecke J. Time of day effects on isometric and isokinetic torque developped during elbow flexions in humans. Eur J Appl Physiol 2001;84:249-52.

[8] Hagberg JM, Mullin JP, Giese MD, Spitznagel E. Effect of pedaling rate on submaximal exercise responses of competitive cyclists. J Appl Physiol 1981;51(2):447-51. 
[9] Hausswirth C, Brisswalter J, Vallier JM, Smith D, Lepers R. Evolution of electromyographic signal, running economy, and perceived exertion during different prolonged exercises. Int J Sports Med 2000;21(6):42936.

[10] Howley ET, Bassett Jr. DR, Welch HG. Criteria for maximal oxygen uptake: review and commentary. Med Sci Sports Exerc 1995;27(9): 1292-301.

[11] Lepers R, Hausswirth C, Maffiuletti NA, Brisswalter J, Van Hoecke J. Evidence of neuromuscular fatigue after prolonged cycling exercice. Med Sci Sports Exerc 2000;32:1880-6.

[12] Lucia A, Hoyos J, Chicharro JL. Preferred pedalling cadence in professional cycling. Med Sci Sports Exerc 2001;33(8):1361-6.

[13] MacIntosh BR, Neptune RR, Horto JF. Cadence, power, and muscle activation in cycle ergometry. Med Sci Sports Exerc 2000;32(7):1281-7.

[14] Marsh AP, Martin PE. The association between cycling experience and preferred and most economical cadences. Med Sci Sports Exerc 1993;25 (11):1269-74.

[15] Marsh AP, Martin PE. Effect of cycling experience, aerobic power, and power output on preferred and most economical cycling cadences. Med Sci Sports Exerc 1997;29(9):1225-32.

[16] Marsh AP, Martin PE, Foley KO. Effect of cadence, cycling experience, and aerobic power on delta efficiency during cycling. Med Sci Sports Exerc 2000;32(9):1630-4.

[17] Méro A, Häkkinen K, Kauhanen H. Hormonal profile and strength development in young weight lifters. J Hum Mov Stud 1989;16:255-66.

[18] Neptune RR, Hull ML. A theoretical analysis of preferred pedaling rate selection in endurance cycling. J Biomech 1999;32(4):409-15.

[19] Neptune RR, Van Den Bogert AJ. Standard mechanical energy analyses do not correlate with muscle work in cycling. J Biomech 1998;31:23945.

[20] Pandolf KB, Noble BJ. The effect of pedaling speed and resistance changes on perceived exertion for equivalent power outputs on the bicycle ergometer. Med Sci Sports Exerc 1973;5:132-6.

[21] Patterson RP, Moreno MI. Bicycle pedalling forces as a function of pedalling rate and power output. Med Sci Sports Exerc 1990;22(4):5126.
[22] Pugh LG. The relation of oxygen intake and speed in competition cycling and comparative observations on the bicycle ergometer. J Physiol 1974;241:795-808.

[23] Reilly T, Down A. Investigation of circadian rythms in anaerobic power and capacity of the legs. J Sports Med Phys Fitness 1992;32:343-7.

[24] Reinhard U, Muller PH, Schmulling RM. Determination of anaerobic threshold by the ventilation equivalent in normal individuals. Respiration (Herrlisheim) 1979;38(1):36-42.

[25] Sargeant AJ. Human power output and muscle fatigue. Int J Sports Med 1994;15(3):116-21.

[26] Sarre G, Lepers R, Maffiuletti N, Millet G, Martin A. Influence of cycling cadence on neuromuscular activity of the knee extensors in humans. Eur J Appl Physiol 2003;88(4-5):476-9.

[27] Seabury JJ, Adams WC, Ramey MR. Influence of pedalling rate and power output on energy expenditure during bicycle ergometry. Ergonomics 1977;20:491-8.

[28] Stone MH, O’Bryant HS. Weight training: a scientific approach. Minneapolis: Burgess Publisching; 1987.

[29] Suzuki Y. Mechanical efficiency of fast and slow twitch muscles fibers in man during cycling. J Appl Physiol 1979;47(2):263-7.

[30] Takaishi T, Yasuda Y, Ono T, Moritani T. Optimal pedaling rate estimated from neuromuscular fatigue for cyclists. Med Sci Sports Exerc 1996; 28(12):1492-7.

[31] Takaishi T, Yamamoto T, Ono T, Ito T, Moritani T. Neuromuscular, metabolic, and kinetic adaptations for skilled pedaling performance in cyclists. Med Sci Sports Exerc 1998;30(3):442-9.

[32] Vercruyssen F, Hausswirth C, Smith D, Brisswalter J. Effect of exercise duration on optimal pedaling rate choice in triathletes. Can J Appl Physiol 2001;26(1):44-54.

[33] Vercruyssen F, Brisswalter J, Hausswirth C, Bernard T, Bernard O, Vallier JM. Influence of cycling cadence on subsequent running performance in triathletes. Med Sci Sports Exerc 2002;34(3):530-6.

[34] Vercruyssen F, Suriano R, Bishop D, Hausswirth C, Brisswalter J. Cadence selection affects metabolic responses during cycling and subsequent running time to fatigue. Br J Sports Med 2005;39(5):267-72.

[35] Vollestad NK, Blom PC. Effect of varying exercise intensity on glycogen depletion in human muscle fibres. Acta Physiol Scand 1985;125(3):395405. 